\title{
Self-Propulsion of Active Colloids via Ion Release: Theory and Experiments
}

\author{
Marco De Corato $\odot^{*}$ and Xavier Arqué \\ Institute for Bioengineering of Catalonia (IBEC), The Barcelona Institute of Science and Technology (BIST), \\ Baldiri Reixac 10-12, 08028 Barcelona Spain \\ Tania Patiño \\ Institute for Bioengineering of Catalonia (IBEC), The Barcelona Institute of Science and Technology (BIST), \\ Baldiri Reixac 10-12, 08028 Barcelona Spain \\ Chemistry Department, University of Rome, Tor Vergata, Via della Ricerca Scientifica, 00133 Rome, Italy \\ Marino Arroyo \\ LaCàN, Universitat Politècnica de Catalunya-BarcelonaTech, 08034 Barcelona, Spain; \\ Institute for Bioengineering of Catalonia (IBEC), The Barcelona Institute of Science and Technology (BIST), \\ Baldiri Reixac 10-12, 08028 Barcelona, Spain; \\ and Centre Internacional de Mètodes Numèrics en Enginyeria (CIMNE), 08034 Barcelona, Spain \\ Samuel Sánchez \\ Institute for Bioengineering of Catalonia (IBEC), The Barcelona Institute of Science and Technology (BIST), \\ Baldiri Reixac 10-12, 08028 Barcelona Spain; \\ and Institució Catalana de Recerca i Estudis Avançats (ICREA), Pg. Lluís Companys 23, 08010 Barcelona, Spain \\ Ignacio Pagonabarraga® \\ Departament de Física de la Matèria Condensada, Universitat de Barcelona, C. Martí Franquès 1, 08028 Barcelona, Spain; \\ University of Barcelona Institute of Complex Systems (UBICS), Universitat de Barcelona, 08028 Barcelona, Spain; \\ and CECAM, Centre Européen de Calcul Atomique et Moléculaire, École Polytechnique Fédérale de Lasuanne (EPFL), \\ Batochime, Avenue Forel 2,1015 Lausanne, Switzerland
}

(Received 11 December 2019; accepted 11 February 2020; published 13 March 2020)

\begin{abstract}
We study the self-propulsion of a charged colloidal particle that releases ionic species using theory and experiments. We relax the assumptions of thin Debye length and weak nonequilibrium effects assumed in classical phoretic models. This leads to a number of unexpected features that cannot be rationalized considering the classic phoretic framework: an active particle can reverse the direction of motion by increasing the rate of ion release and can propel even with zero surface charge. Our theory predicts that there are optimal conditions for self-propulsion and a novel regime in which the velocity is insensitive to the background electrolyte concentration. The theoretical results quantitatively capture the salt-dependent velocity measured in our experiments using active colloids that propel by decomposing urea via a surface enzymatic reaction.
\end{abstract}

DOI: $10.1103 /$ PhysRevLett.124.108001

To overcome random thermal fluctuations, microscopic active objects, such as bacteria [1] and molecular motors [2], convert chemical energy into motion. Inspired by nature, there has been an extensive effort in developing biomimetic synthetic active particles that employ catalytic chemical reactions to self-propel $[3,4]$. Suspensions of these colloid-sized particles have been used as a model to investigate nonequilibrium active systems [5-8], ordering and phase transitions $[9,10]$, and collective communication [11-13]. Functionalized active colloids are also potential candidates for novel cancer therapies [14,15], cargo transport [16], or other biomedical applications $[17,18]$. In order to control the dynamics of active particles, whether for technological applications or to study their collective motion, one has to understand the mechanism that couples their chemical activity to motion.

Chemically active particles with a number of different propulsion mechanisms have been considered [19-25]. They rely on establishing a gradient of chemical potential along the surface of the particle, which drives its motion via phoretic flows [26,27]. Typically, the gradient of chemical potential is introduced by an asymmetric chemical activity that sustains gradients of solutes. For active colloids propelling by the generation of ionic species, the classic theory of phoresis [26,28] has been widely applied [29-35]. However, the theory of phoresis was developed to study the motion of colloids in weak gradients of electrolytes [28] and thin Debye layers [36]. It seems natural to ask whether 
the same framework is applicable to chemically active particles, which can be driven far from equilibrium or display a thick Debye layer.

To address this question, we investigate a general model for a charged active colloid that propels by releasing ionic species. We find that the surface flux of ions leads to a strong coupling between the solute transport and the charge balance in which the different diffusivity of positive and negative ions plays a fundamental role. Such coupling results in behaviors that cannot be explained using the standard phoretic models [26,28], which break down for active colloids that release ions at large rates. To demonstrate the relevance of the model, we compare its predictions with our measurements of the velocity of enzyme-powered colloids that propel by decomposing urea into ammonia and carbon dioxide.

Consider a system containing a spherical nonconductive particle of radius $R$ that is homogeneously charged with charge density $q_{s}$ and is suspended in an electrolyte solution. We assume that the electrolyte is a salt with unit valence and number density $n_{\infty}$, which is dissolved in a solvent with shear viscosity $\eta$ and density $\rho$. The particle is chemically active and releases ions from a portion of its surface with a surface flux $Q$, which we assume axisymmetric. We denote the excess number density of the cation with $c_{+}$and the excess number density of the anion with $c_{-}$, with their diffusivity given by $D_{+}$and $D_{-}$. See Fig. 1 for an illustration. The model is applicable to cases where ions are released by chemical reactions or by dissolving salt particles, in the limit of irreversible decomposition of neutral species into ions. The asymmetric chemical activity of the particle drives its motion at a velocity $V \boldsymbol{e}_{z}$, with $\boldsymbol{e}_{z}$ the unit vector along the $z$ axis. The ionic species released by the particle drive the system out of equilibrium, introducing an additional charge density and electric field, whose interaction results in an asymmetric electrostatic force

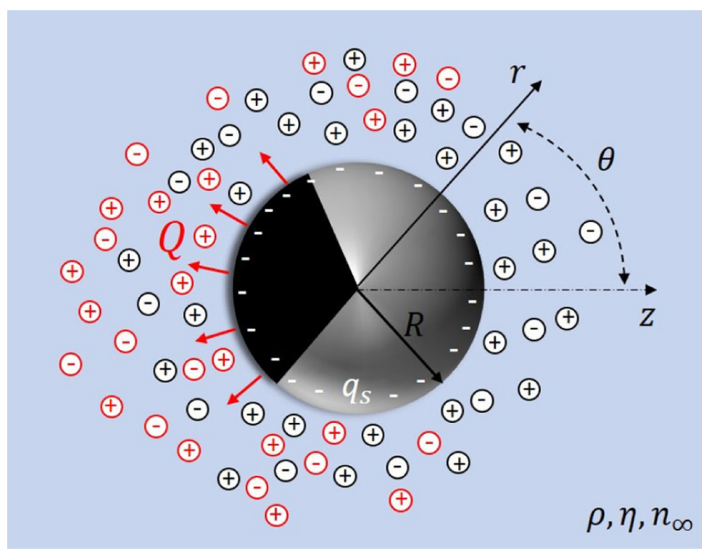

FIG. 1. A nonconductive particle with surface charge density $q_{s}$ is suspended in an electrolyte solution, of number density $n_{\infty}$, and releases ions from a portion of its surface with a surface flux $Q$. The ions already in solution are displayed in black, the excess ions in red. density that propels the particle. Here we neglect thermal fluctuations, which can be relevant in the case of selfpropelled nanoparticles [37-39].

To evaluate the velocity of the active particle, we consider the balance of number density of the excess ionic species rescaled with $n_{\infty}$. We assume that the species are dilute and that the system is at steady state. We further neglect advection as the Peclet number, $\mathrm{Pe}=V R / D_{ \pm}$, quantifying the importance of advection compared to diffusion, is much smaller than one for colloidal particles [40]. Under these assumptions, the dimensionless balance of number density reads

$$
\boldsymbol{\nabla}^{2} c_{ \pm} \pm \boldsymbol{\nabla} \cdot\left(c_{ \pm} \boldsymbol{\nabla} \Phi\right)=0
$$

with the dimensionless electric potential $\Phi$ scaled with the elementary charge $e$ and the thermal energy $k_{B} T$. The excess ionic species vanish at infinity and are produced at the surface with a dimensionless flux, $\left(\nabla c_{+}+c_{+} \nabla \Phi\right)$. $\boldsymbol{n}=Q^{*} f(\theta) \quad$ and $\quad\left(\boldsymbol{\nabla} c_{-}-c_{-} \boldsymbol{\nabla} \Phi\right) \cdot \boldsymbol{n}=\beta^{-1} Q^{*} f(\theta)$, which applies at $r=1$. $\beta=D_{-} / D_{+}$denotes the ratio of diffusivity and $Q^{*}=Q R / D_{+} n_{\infty}$ controls the ratio of the characteristic excess number density to the number density of the ionic species already in the solution. When the surface flux is small, $Q^{*} \ll 1$, the number density of the excess ionic species is much smaller than $n_{\infty}$ and the system is close to equilibrium. Conversely, when $Q^{*} \gg 1$, the number density of the excess ionic species is much larger than $n_{\infty}$ and the system is significantly out of equilibrium. The function $f(\theta)$ determines the distribution of the surface chemical activity and can be decomposed in Legendre polynomials $f(\theta)=\sum_{l=0}^{\infty} f_{l} P_{l}(\cos \theta)$, with $f_{l}$ its multipole moment.

The ionic species already dissolved in the solution are at equilibrium with respect to the electric potential. Their distribution is given by the Boltzmann distribution $n_{\infty} \exp \left( \pm e \Phi / k_{B} T\right)$. Therefore, the dimensionless balance of charge density in the fluid phase satisfies a modified Poisson-Boltzmann equation:

$$
\boldsymbol{\nabla}^{2} \Phi=\frac{\kappa^{2}}{2}\left(c_{-}-c_{+}\right)+\kappa^{2} \sinh (\Phi)
$$

with $\kappa$ the inverse of the Debye length, normalized by the particle radius, $\kappa=\sqrt{\left(2 e^{2} n_{\infty} R^{2} / k_{B} T \epsilon\right)}$, with the permittivity of the fluid $\epsilon$. We consider zero electric potential at infinity, and a uniform surface charge density, $-\nabla \Phi \cdot \boldsymbol{n}=$ $q_{s}^{*}$, with $q_{s}^{*}=q_{s} \operatorname{Re} / k_{B} T \epsilon$.

For small Reynolds numbers, $\operatorname{Re}=\rho V R / \eta \ll 1$, the velocity of the particle, made dimensionless with $e^{2} \eta R /\left(k_{B} T\right)^{2} \epsilon$, is calculated using the reciprocal theorem, which involves an integral over the fluid volume $\Omega$ outside the particle $[40,47,48]$ :

$$
V=-\frac{\kappa^{2}}{12 \pi} \int_{\Omega} \boldsymbol{f} \cdot\left(\hat{\boldsymbol{v}}-\boldsymbol{e}_{z}\right) d \Omega,
$$


with $\hat{v}$ the velocity field around a sphere translating with unit velocity along $\boldsymbol{e}_{z}$ and the electrostatic force density $\boldsymbol{f}=\left[c_{+}-c_{-}-2 \sinh (\Phi)\right] \boldsymbol{\nabla} \Phi$. The propulsion velocity is a function of four dimensionless numbers: $V=V\left(q_{s}^{*}\right.$, $\left.Q^{*}, \kappa, \beta\right)$. We investigate next its functional dependence on the dimensionless numbers using asymptotic analysis and numerical simulations.

Particle velocity near equilibrium in the Debye-Hückel limit.-To investigate the mechanisms leading to selfpropulsion at arbitrary $\kappa$, we solve the governing equations in the limit of small $Q^{*}$ and $q_{s}^{*}$ using a regular perturbation expansion [40]. We consider the case of a monopolar and dipolar surface flux, $f_{l}=0$ for $l>1$. We find that the leading-order velocity is

$V=Q^{*^{2}} \frac{f_{0} f_{1}(1-\beta)^{2}}{\beta^{2}} g(\kappa)+Q^{*} q_{s}^{*} \frac{f_{1}(1-\beta)}{\beta} h(\kappa)+$ hot,

which is valid for arbitrary values of $\kappa$. The positive functions $g(\kappa)$ and $h(\kappa)$ encode the dependence on the dimensionless Debye length and are reported in the Supplemental Material [40]. By setting the monopolar term to zero, $f_{0}=0$, Eq. (4) recovers the velocity found by Brown et al. [49], who considered a dipolar ion release only. In the case $\beta=1$, the leading-order terms given by Eq. (4) vanish, and the velocity is proportional to higherorder terms. The well-known chemiphoretic contribution for equal ionic diffusivity does not appear in Eq. (4) because it depends nonlinearly on the surface charge density [28]. To assess the validity of the asymptotic theory, we tested Eq. (4) using numerical simulations [40].

The second term in the right-hand side of Eq. (4) stems from the interaction of the charge density generated by the excess ions with the equilibrium electric potential. It represents the standard phoretic contribution at small surface charge densities and predicts a velocity whose sign depends on $q_{s}^{*}(1-\beta)$. For thin Debye layers, $\kappa \gg 1$, this term recovers the velocity predicted using the framework of Prieve et al. [28]. Conversely, the first term on the righthand side of Eq. (4) was overlooked by previous works
$[31,34,49]$ and shows that the particle propels even if not charged, $q_{s}^{*}=0$. This mechanism is fundamentally different from the standard phoretic mechanisms that rely on the existence of an interaction potential between the solute molecules and the surface of the particle $[26,28,50]$. Here, the motion of the particle originates entirely from the surface flux of ions, which couples transport of species and the balance of charges. This mechanism is relevant when $\beta \neq 1$, which is usually the case in experiments [51], and highlights the importance of considering the different ionic diffusivities in out-of-equilibrium systems. The first term in Eq. (4) is always positive and leads to motion toward the portion of the surface having the largest flux magnitude.

If the two terms in Eq. (4) have opposite signs, the direction of motion is determined by the prefactors and by the functions $g(\kappa)$ and $h(\kappa)$. For large Debye lengths, $\kappa \ll 1$, we find $g \propto \kappa^{4}$ and $h \propto \kappa^{2}$. In this limit, the second term in Eq. (4) dominates over the first one and the direction of motion is determined by $Q^{*} q_{s}^{*}(\beta-1)$. In the case $\kappa \gg 1$, we find the asymptotic behavior $g(\kappa) \propto \kappa^{-1}$ and $h(\kappa) \propto \kappa^{-1}$, with the two functions having the same order of magnitude, $\lim _{\kappa \rightarrow \infty} h(\kappa) / g(\kappa)=2$ [40]. It is thus possible to reverse the direction of motion by changing the salt concentration, the particle size, or the surface flux, as observed in the experiments of Brown and Poon [52].

Numerical results for a Janus particle.-In many practical situations the flux of ionic species and the surface charge can be large and nonlinear effects become dominant. We investigate the velocity of a particle at nonvanishing $Q^{*}$ and $q_{s}^{*}$ using finite element simulations [40]. We assume that half of the surface of the particle releases ionic species: $f(\theta)=1$ for $\theta \leq(\pi / 2), f(\theta)=0$ for $\theta>(\pi / 2)$, and $Q^{*}>0$.

Recent works $[30,33,34]$ applied the framework of ionic phoresis [28], which assumes a thin Debye layer $\kappa \gg 1$, to study chemically active particles. It is not clear to what extent the theory developed for weak external gradients of ionic species can be applied to chemically active particles far from equilibrium. To address this point, we compare in Fig. 2(a) the velocity predicted by Eqs. (1)-(3) to that predicted using the model of Prieve et al. [28]; see also Ref. [40]. We consider unequal ion diffusivity $\beta=2$ and a
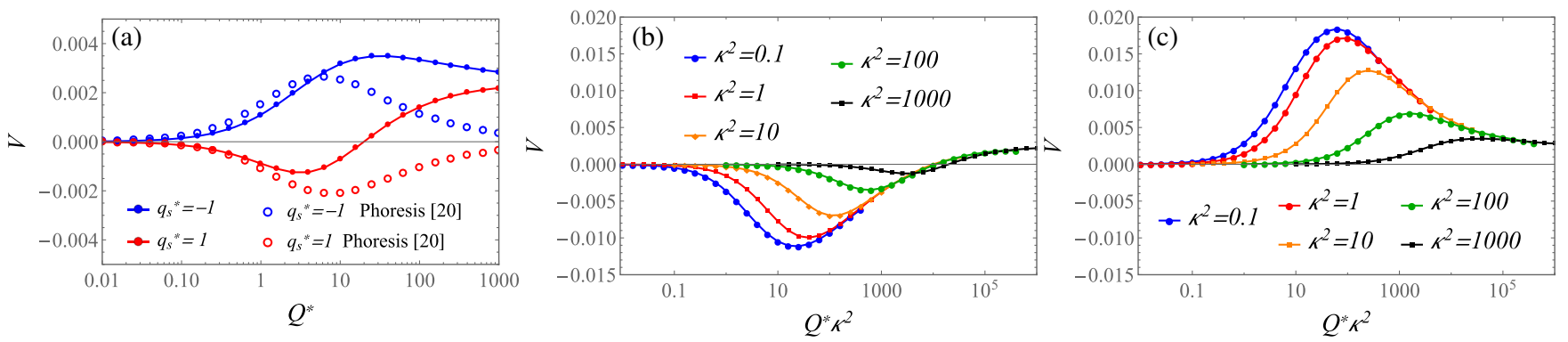

FIG. 2. (a) Velocity predicted by our theory compared to the predictions of the standard ionic phoretic model $[28,40]$ in the case $\kappa^{2}=1000$ and $\beta=2$. (b) Velocity computed using simulations for the case $\beta=2$ and $q_{s}^{*}=1$. (c) Dimensionless velocity computed using simulations for the case $\beta=2$ and $q_{s}^{*}=-1$. For $Q^{*} \gg \kappa^{2}$, the velocity curves at different $\kappa$ collapse onto a master curve. In this regime, the velocity is independent from the salt concentration. 
thin Debye length $\kappa^{2}=1000$; we expect the model of Prieve et al. [28] to be valid for these parameters. Instead, Fig. 2(a) shows that the standard ionic phoretic model breaks down for systems driven sufficiently far from equilibrium, $Q^{*} \approx 1$, and, for $Q^{*} \gg 1$, it can fail to predict the correct direction of motion. We further discuss the deviation between the two models in the Supplemental Material [40].

In Figs. 2(b) and 2(c) we show that the functional dependence of $V$ on $Q^{*}$ can be divided into three regimes. We find a linear regime at small surface fluxes, $Q^{*} \ll 1$, where the standard phoretic mechanisms [26] are dominant and $V \propto Q^{*}$ with a slope that depends on $q_{s}^{*}$ and $\kappa$. In the case $Q^{*} \gg k^{2}$, we find a large-flux regime in which the velocities at different $\kappa$ collapse onto a master curve and become a function of $Q^{*} \kappa^{2}=2 Q R^{3} e^{2} / D_{+} k_{B} T \epsilon$. This dimensionless group is independent from the background electrolyte concentration $n_{\infty}$. In this regime, the propulsion velocity is independent of $n_{\infty}$ because the number density of the excess ionic species is much larger than $n_{\infty}$. Further, in the limit $Q^{*} \kappa^{2} \rightarrow \infty$, the velocity reaches a plateau that depends weakly on the surface charge. This is an interesting regime for practical applications because the propulsion velocity is insensitive to the background electrolyte concentration and to the surface charge of the particle. Most of the charge density in the fluid is generated by the excess ionic species, and the surface charge of the particle plays a minor role. At intermediate values of $Q^{*}$ the magnitude of the velocity goes through a maximum, indicating that there is an optimal value of $Q^{*}$, which depends on the system parameters. Finally, we find that the qualitative conclusions drawn from the asymptotic theory, Eq. (4), hold beyond the leading order. This is illustrated in Fig. 2(b), where the velocity for $\beta=2$ appears to change sign at a critical $Q^{*}$, as predicted by Eq. (4) in the case $q_{s}^{*}(1-\beta)<0$. Conversely, in the case $q_{s}^{*}(1-\beta)>0$ displayed in Fig. 2(c), the velocity is always positive as predicted by Eq. (4).

Comparison with experiments using enzyme-powered colloids. - We compare the predictions of the model with velocity measurements of chemically active colloidal particles, $R=1 \mu \mathrm{m}$. We perform experiments using functionalized particles that propel via the enzymatic decomposition of urea $\mathrm{CO}\left(\mathrm{NH}_{2}\right)_{2}$ into ammonia $\mathrm{NH}_{3}$ and carbon dioxide $\mathrm{CO}_{2}$, catalyzed by urease tethered to their surface $[40,53]$. The products form ions when dissolved in water according to the equilibria $\mathrm{NH}_{3}+\mathrm{H}_{2} \mathrm{O} \rightleftharpoons \mathrm{NH}_{4}^{+}+\mathrm{OH}^{-}$and $\mathrm{CO}_{2}+$ $\mathrm{H}_{2} \mathrm{O} \rightleftharpoons \mathrm{HCO}_{3}^{-}+\mathrm{H}^{+}$. Recent works reported a local [54] and global [14] $p \mathrm{H}$ increase when urea is decomposed by the urease-powered colloids. This suggests that most of the ionic species are introduced by the reaction of $\mathrm{NH}_{3}$ with water. To employ the theoretical framework developed here, we assume that the ammonia produced by urease turns instantaneously and irreversibly into $\mathrm{NH}_{4}^{+}$ and $\mathrm{OH}^{-}$and that these excess ionic species drive the motion of the particle. To test the theoretical predictions, we study the effect of the background salt concentration on the velocity of the particles.

The parameters of the model can be measured or estimated from the experimental conditions. The ratio of the diffusion coefficients of $\mathrm{NH}_{4}^{+}$and $\mathrm{OH}^{-}$is known and yields $\beta=2.7$ [55]. The velocity of the colloids is measured at $200 \mathrm{mM}$ urea in ultrapure water and highly diluted phosphate-buffered saline solutions (PBS) with a maximum dilution ratio of $1: 250$. Since $90 \%$ of PBS is made of the monovalent electrolyte $\mathrm{NaCl}$, we assume that PBS contains $\mathrm{NaCl}$ only. We vary the background electrolyte concentration $n_{\infty}$ over 4 orders of magnitude: from $n_{\infty}=0.1 \mu \mathrm{M}$, in ultrapure water, to $n_{\infty}=5000 \mu \mathrm{M}$ in $1: 250$ PBS solutions. Since the concentration of urea is much larger than the Michaelis-Menten constant, $K_{m} \approx$ 1-10 mM [56,57], the enzymes work at their maximum rate and the reaction rate is independent of the local urea concentration. We estimate the surface flux of ionic species as $Q=k_{\text {cat }} \rho_{\text {ur }}$, with the turnover rate $k_{\text {cat }} \approx 10^{4} \mathrm{~s}^{-1}$ estimated from previous works measuring ammonia production [58,59], and the surface density of enzymes $\rho_{\text {ur }}=10^{16} \mathrm{~m}^{-2}$ estimated from our previous work where we used stochastic optical reconstruction microscopy [60]. The spherical symmetry of the surface flux is broken by the presence of imperfections of the particle surface (see Fig. 3 in Supplemental Material [40]) and by an

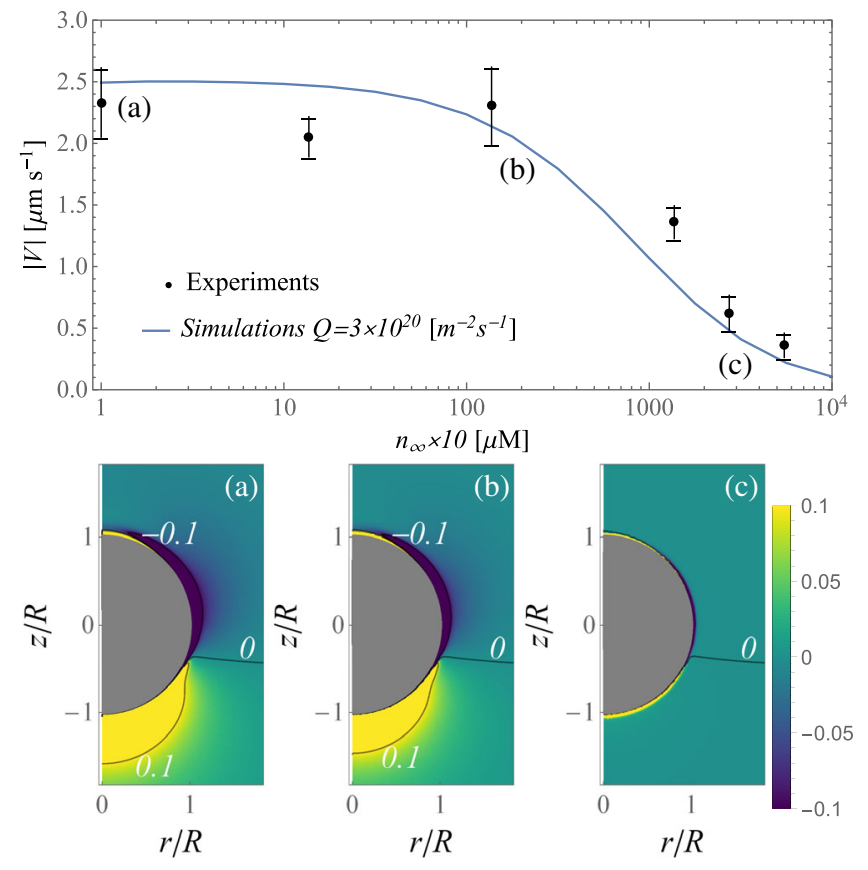

FIG. 3. Top: Comparison between the magnitude of the velocity predicted by the simulations and our measurements in experiments at different background electrolyte concentration $n_{\infty}$. Error bars represents the standard error of the mean. Bottom: (a)-(c) Density and contour plots of the $z$ component of the dimensionless electrostatic force density $f$ obtained from the simulations. Each plot corresponds to the case marked with the same letter in the top panel. 
asymmetric enzyme attachment to the silica surface that forms patches [60]. We assume that these features can be captured by a monopolar and dipolar surface flux $f_{0}=$ $f_{1}=1$ yielding a flux distribution $f(\theta)=1+\cos (\theta)$. Electrophoretic measurements yield a surface charge density $q_{s}=-16 \mu \mathrm{Cm}^{-2}$; see Fig. 4 in Ref. [40]. The experiments range from a strongly nonequilibrium regime $Q^{*} \approx 3000$ and thick Debye layer $\kappa \approx 1$ in ultrapure water to a near-equilibrium regime $Q^{*} \approx 0.5$ and a thin Debye layer $\kappa \approx 150$ in 1:250 PBS solutions; see Fig. 5 in Ref. [40].

The top panel of Fig. 3 shows that there is a good agreement between the velocity measured in the experiments and that predicted by the model for a flux that is comparable to that expected from our estimate. Remarkably, at small $n_{\infty}$, the experiments confirm the existence of plateau where the velocity is independent from the background salt concentration as predicted by the simulations; see Figs. 2(b) and 2(c). The plateau is observed in a regime where $Q^{*} \approx$ 3000 and $\kappa \approx 1$ and, therefore, $Q^{*} \gg \kappa^{2}$, in which nonequilibrium effects are important. In the bottom panel of 3 we plot the axial component of the electrostatic force density $f$ around the active colloid. According to phoretic models [26], the electrostatic force density should be confined to a thin layer next to the particle surface. In fact, Figs. 3(a) and 3(b) show that far from equilibrium $\boldsymbol{f}$ is spread around the particle. The breakdown of phoretic models is particularly evident in the case of Fig. 3(b) in which $\kappa \approx 20$, and one would expect $f$ to decay rapidly away from the colloid surface, which is not the case if $Q^{*} \gg 1$. As $n_{\infty}$ is increased, the number density of the background ions becomes comparable to the excess ions released by the particle and the velocity decreases. In this regime, Fig. 3(c) shows that the electrostatic force density is concentrated within a thin boundary layer as assumed in phoretic models.

Conclusions. - We have shown that the nonequilibrium nature of active colloids that release ions can lead to qualitatively different behaviors compared to standard phoretic mechanisms. The features we identified are relevant when the diffusion coefficients of the ionic species are different and cannot be explained using the classic ionic phoretic approaches, which break down if the particle is significantly driven out of equilibrium by its chemical activity. The predictions quantitatively explain the dependence of the velocity of urease-powered colloids on the background concentration of electrolytes measured in our experiments, thus confirming that the regimes investigated here are relevant for experiments in electrolyte solutions. The effects discovered here shed light on the mechanisms behind the propulsion of active colloids far from equilibrium. Exploiting the new phenomena discussed could open new possibilities for engineering their cooperative motion and for tuning their interactions with confining surfaces.

M. D. C. acknowledges funding from the European Union's Horizon 2020 research and innovation program under the Marie Skłodowska-Curie action (GA 712754), the Severo Ochoa programme (SEV-2014-0425), and the CERCA Programme/Generalitat de Catalunya. I. P. acknowledges support from MINECO Project No. PGC2018-098373B-I00, DURSI Project No. 2017SGR-884, and SNF Project No. 200021-175719. M. A. acknowledges the support from the Generalitat de Catalunya (ICREA Academia Award) and from the Spanish Ministry of Economy and Competitiveness, through the Severo Ochoa Programme (CEX2018-000797S). M. D. C. and I. P. acknowledge funding from the H2020 research and innovation program under the FET open project NanoPhlow. X. A. thanks MINECO for the Severo Ochoa programme (SEV-2014-0425) for the financial support of the Ph.D. fellowship (PRE2018-083712). T. P. thanks MINECO for the support of the Juan de la Cierva (FJCI-2015-25578) postdoctoral fellowship, and the European Union's Horizon 2020 research and innovation program, under the Marie Skłodowska-Curie Individual Fellowship (H2020-MSCAIF-2018, DNA-bots). S. S. thanks MINECO for the projects MICRODIA (CTQ2015-68879-R), Enzwim (CTQ201572471-EXP), and BOTSinFLUIDS (RTI2018-098164-BI00). S. S. also acknowledges the BBVA Foundation for the MEDIROBOTS project and the CERCA program by the Generalitat de Catalunya. M. D. C. thanks Giovanniantonio Natale and Axel Huerre for their valuable feedback on an earlier version of the manuscript.

*mdecorato@ibecbarcelona.eu

[1] E. Lauga, Annu. Rev. Fluid Mech. 48, 105 (2016).

[2] R. D. Astumian, Science 276, 917 (1997).

[3] X. Ma, A. C. Hortelão, T. Patiño, and S. Sánchez, ACS Nano 10, 9111 (2016).

[4] K. K. Dey and A. Sen, J. Am. Chem. Soc. 139, 7666 (2017).

[5] J. Palacci, C. Cottin-Bizonne, C. Ybert, and L. Bocquet, Phys. Rev. Lett. 105, 088304 (2010).

[6] J. Palacci, S. Sacanna, A. P. Steinberg, D. J. Pine, and P. M. Chaikin, Science 339, 936 (2013).

[7] C. Bechinger, R. Di Leonardo, H. Löwen, C. Reichhardt, G. Volpe, and G. Volpe, Rev. Mod. Phys. 88, 045006 (2016).

[8] S. C. Takatori, R. De Dier, J. Vermant, and J. F. Brady, Nat. Commun. 7, 10694 (2016).

[9] M. C. Marchetti, J.-F. Joanny, S. Ramaswamy, T. B. Liverpool, J. Prost, M. Rao, and R. A. Simha, Rev. Mod. Phys. 85, 1143 (2013).

[10] F. Ginot, I. Theurkauff, F. Detcheverry, C. Ybert, and C. Cottin-Bizonne, Nat. Commun. 9, 696 (2018).

[11] U. Khadka, V. Holubec, H. Yang, and F. Cichos, Nat. Commun. 9, 3864 (2018).

[12] J. Agudo-Canalejo and R. Golestanian, Phys. Rev. Lett. 123, 018101 (2019).

[13] F. A. Lavergne, H. Wendehenne, T. Bäuerle, and C. Bechinger, Science 364, 70 (2019).

[14] A. C. Hortelão, T. Patiño, A. Perez-Jiménez, A. Blanco, and S. Sánchez, Adv. Funct. Mater. 28, 1705086 (2018).

[15] A. C. Hortelão, R. Carrascosa, N. Murillo-Cremaes, T. Patiño, and S. Sánchez, ACS Nano 13, 429 (2019). 
[16] J. Palacci, S. Sacanna, A. Vatchinsky, P. M. Chaikin, and D. J. Pine, J. Am. Chem. Soc. 135, 15978 (2013).

[17] J. Li, S. Thamphiwatana, W. Liu, B. Esteban-Fernéndez de Àvila, P. Angsantikul, E. Sandraz, J. Wang, T. Xu, F. Soto, V. Ramez et al., ACS Nano 10, 9536 (2016).

[18] Z. Wu, J. Troll, H.-H. Jeong, Q. Wei, M. Stang, F. Ziemssen, Z. Wang, M. Dong, S. Schnichels, T. Qiu et al., Sci. Adv. 4, eaat4388 (2018).

[19] W. F. Paxton, K. C. Kistler, C. C. Olmeda, A. Sen, S. K. St. Angelo, Y. Cao, T. E. Mallouk, P. E. Lammert, and V. H. Crespi, J. Am. Chem. Soc. 126, 13424 (2004).

[20] S. J. Ebbens and J. R. Howse, Soft Matter 6, 726 (2010).

[21] I. Buttinoni, G. Volpe, F. Kümmel, G. Volpe, and C. Bechinger, J. Phys. Condens. Matter 24, 284129 (2012).

[22] Z. Izri, M. N. van der Linden, S. Michelin, and O. Dauchot, Phys. Rev. Lett. 113, 248302 (2014).

[23] K. K. Dey, X. Zhao, B. M. Tansi, W. J. Méndez-Ortiz, U. M. Córdova-Figueroa, R. Golestanian, and A. Sen, Nano Lett. 15, 8311 (2015).

[24] J. Katuri, X. Ma, M. M. Stanton, and S. Sánchez, Acc. Chem. Res. 50, 2 (2017).

[25] E. O’Neel-Judy, D. Nicholls, J. Castañeda, and J. G. Gibbs, Small 14, 1801860 (2018).

[26] J. L. Anderson, Annu. Rev. Fluid Mech. 21, 61 (1989).

[27] R. B. Bird, W. E. Stewart, and E. N. Lightfoot, Transport Phenomena (John Wiley \& Sons, New York, 2007).

[28] D. Prieve, J. Anderson, J. Ebel, and M. Lowell, J. Fluid Mech. 148, 247 (1984).

[29] W. Duan, M. Ibele, R. Liu, and A. Sen, Eur. Phys. J. E 35, 77 (2012).

[30] W. Duan, R. Liu, and A. Sen, J. Am. Chem. Soc. 135, 1280 (2013).

[31] P. Bayati and A. Najafi, J. Chem. Phys. 144, 134901 (2016).

[32] X. Wang, L. Baraban, A. Nguyen, J. Ge, V. R. Misko, J. Tempere, F. Nori, P. Formanek, T. Huang, G. Cuniberti, J. Fassbender, and D. Makarov, Small 14, 1803613 (2018).

[33] C. Zhou, H. Zhang, J. Tang, and W. Wang, Langmuir 34, 3289 (2018).

[34] F. Yang, B. Rallabandi, and H. A. Stone, J. Fluid Mech. 865, 440 (2019).

[35] P. Bayati and A. Najafi, J. Chem. Phys. 150, 234902 (2019).

[36] J. N. Israelachvili, Intermolecular and Surface Forces (Academic Press, Amsterdam, 2015).

[37] R. Golestanian, Phys. Rev. Lett. 102, 188305 (2009).

[38] A. Mozaffari, N. Sharifi-Mood, J. Koplik, and C. Maldarelli, Phys. Rev. Fluids 3, 014104 (2018).
[39] S.-Y. Reigh, M.-J. Huang, H. Löwen, E. Lauga, and R. Kapral, Soft Matter 16, 1236 (2020).

[40] See Supplemental Material at http://link.aps.org/ supplemental/10.1103/PhysRevLett.124.108001 for details on the analytical solution, the numerical method, the comparison with the standard phoretic model, the experiments; supplementary Figs. S1-S5, which includes Refs. [28,34,41-46].

[41] S. Michelin and E. Lauga, J. Fluid Mech. 747, 572 (2014).

[42] E. Yariv, Proc. R. Soc. A 467, 1645 (2011).

[43] E. Hinch, Perturbation Methods (Cambridge University Press, Cambridge, England, 1991).

[44] K. Makino and H. Ohshima, Langmuir 26, 18016 (2010).

[45] J. R. Howse, R. A. L. Jones, A. J. Ryan, T. Gough, R. Vafabakhsh, and R. Golestanian, Phys. Rev. Lett. 99, 048102 (2007).

[46] G. Dunderdale, S. Ebbens, P. Fairclough, and J. Howse, Langmuir 28, 10997 (2012).

[47] L. G. Leal, Advanced Transport Phenomena: Fluid Mechanics and Convective Transport Processes, Vol. 7 (Cambridge University Press, Cambridge, England, 2007).

[48] H. Masoud and H. A. Stone, J. Fluid Mech. 879, P1 (2019).

[49] A. T. Brown, W. C. Poon, C. Holm, and J. de Graaf, Soft Matter 13, 1200 (2017).

[50] J. L. Moran and J. D. Posner, Annu. Rev. Fluid Mech. 49, 511 (2017).

[51] D. Velegol, A. Garg, R. Guha, A. Kar, and M. Kumar, Soft Matter 12, 4686 (2016).

[52] A. Brown and W. Poon, Soft Matter 10, 4016 (2014).

[53] X. Arqué, A. Romero-Rivera, F. Feixas, T. Patiño, S. Osuna, and S. Sánchez, Nat. Commun. 10, 2826 (2019).

[54] T. Patiño, A. Porchetta, A. Jannasch, A. Lladó, T. Stumpp, E. Schäffer, F. Ricci, and S. Sánchez, J. Am. Chem. Soc. 25, 7896 (2018).

[55] D. R. Lide, CRC Handbook of Chemistry and Physics, Vol. 85 (CRC Press, Boca Raton, FL, 2004).

[56] K. S. Mangaldas, Y. S. Rajput, and R. Sharma, J. Plant Biochem. Biotechnol. 19, 73 (2010).

[57] L. Berlicki, M. Bochno, A. Grabowiecka, A. Białas, P. Kosikowska, and P. Kafarski, Amino acids 42, 1937 (2012).

[58] R. L. Blakeley, E. C. Webb, and B. Zerner, Biochemistry 8, 1984 (1969).

[59] N. E. Dixon, P. W. Riddles, C. Gazzola, R. L. Blakeley, and B. Zerner, Can. J. Biochem. 58, 1335 (1980).

[60] T. Patiño, N. Feiner-Gracia, X. Arqué, A. Miguel-López, A. Jannasch, T. Stumpp, E. Schäffer, L. Albertazzi, and S. Sánchez, J. Am. Chem. Soc. 140, 7896 (2018). 